\title{
ON THE COMPLETE MOMENT CONVERGENCE OF MOVING AVERAGE PROCESSES GENERATED BY $\rho^{*}$-MIXING SEQUENCES
}

\author{
Mi-Hwa Ko, Tae-Sung Kim, and Dae-Hee Ryu
}

\begin{abstract}
Let $\left\{Y_{i} ;-\infty<i<\infty\right\}$ be a doubly infinite sequence of identically distributed and $\rho^{*}$-mixing random variables with zero means and finite variances and $\left\{a_{i} ;-\infty<i<\infty\right\}$ an absolutely summable sequence of real numbers. In this paper, we prove the complete moment convergence of $\left\{\sum_{k=1}^{n} \sum_{i=-\infty}^{\infty} a_{i+k} Y_{i} / n^{1 / p} ; n \geq 1\right\}$ under some suitable conditions. We extend Theorem 1.1 of Li and Zhang [Y. X. Li and L. X. Zhang, Complete moment convergence of moving average processes under dependence assumptions, Statist. Probab. Lett. 70 (2004), 191-197.] to the $\rho^{*}$-mixing case.
\end{abstract}

\section{Introduction}

We assume that $\left\{Y_{i} ;-\infty<i<\infty\right\}$ is a doubly infinite sequence of identically distributed random variables with zero means and finite variances. Let $\left\{a_{i} ;-\infty<i<\infty\right\}$ be an absolutely summable sequence of real numbers and

$$
X_{k}=\sum_{i=-\infty}^{\infty} a_{i+k} Y_{i}, k \geq 1
$$

Under independence assumptions, i.e., $\left\{Y_{i} ;-\infty<i<\infty\right\}$ is a sequence of independent random variables, many limiting results have been obtained for moving average process $\left\{X_{k} ; k \geq 1\right\}$. For examples, Ibragimov [6] has established the central limit theorem for $\left\{X_{k} ; k \geq 1\right\}$, Burton and Dehling [4] have obtained a large deviation principle for $\left\{X_{k} ; k \geq 1\right\}$ assuming $E \exp \left(t Y_{1}\right)$ $<\infty$ for all $t$, and Li et al. [7] have obtained the following result on complete convergence.

Theorem A. Suppose $\left\{Y_{i} ;-\infty<i<\infty\right\}$ is a sequence of independent and identically distributed random variables. Let $\left\{X_{k} ; k \geq 1\right\}$ be defined as (1.1)

Received February 13, 2008; Revised July 14, 2008.

2000 Mathematics Subject Classification. 60G50,60F15.

Key words and phrases. moving average process, complete moment convergence, $\rho^{*}$ mixing, moment inequality. 
and $1 \leq p<2$. Then $E Y_{1}=0$ and $E\left|Y_{1}\right|^{2 p}<\infty$ imply

$$
\sum_{n=1}^{\infty} P\left\{\left|\sum_{k=1}^{n} X_{k}\right| \geq n^{1 / p} \epsilon\right\}<\infty \text { for all } \epsilon>0 \text {. }
$$

Zhang [12] extended Theorem A to the $\phi$-mixing case and Baek, Kim, and Liang [1] discussed the complete convergence of moving average processes under negative association assumption and Liang [9] obtained some general results on the complete convergence of weighted sums of negatively associated random variables, including moving average processes.

When $\left\{X_{k} ; k \geq 1\right\}$ is a sequence of i.i.d random variables with mean zeros and positive finite variances, Chow [5] obtained the following result on the complete moment convergence:

Theorem B. Suppose that $\left\{X_{k} ; k \geq 1\right\}$ is a sequence of i.i.d random variables with $E X_{1}=0$. For $1 \leq p<2$ and $r>p$, if $E\left\{\left|X_{1}\right|^{r}+\left|X_{1}\right| \log \left(1+\left|X_{1}\right|\right)\right\}<\infty$, then for any $\epsilon>0$, we have

$$
\sum_{n=1}^{\infty} n^{r / p-2-1 / p} E\left\{\left|\sum_{k=1}^{\infty} X_{k}\right|-\epsilon n^{1 / p}\right\}^{+}<\infty .
$$

Recently Li and Zhang [8] showed that this kind of result also holds for moving average processes under negative association as follows:

Theorem C. Suppose $\left\{X_{k} ; k \geq 1\right\}$ is defined as (1.1), where $\left\{a_{i} ;-\infty<i<\right.$ $\infty\}$ is a sequence of real numbers with $\sum_{i=-\infty}^{\infty}\left|a_{i}\right|<\infty$ and $\left\{Y_{i} ;-\infty<i<\right.$ $\infty\}$ is a sequence of identically distributed and negatively associated random variables with $E Y_{1}=0, E Y_{1}^{2}<\infty$. Let $h(x)>0(x>0)$ be a slowly varying function and $1 \leq p<2, r>1+p / 2$. Then $E\left|Y_{1}\right|^{r} h\left(\left|Y_{1}\right|^{p}\right)<\infty$ implies $\sum_{n=1}^{\infty} n^{r / p-2-1 / p} h(n) E\left\{\left|S_{n}\right|-\epsilon n^{1 / p}\right\}^{+}<\infty$, where $S_{n}=\sum_{k=1}^{n} X_{k}, n \geq 1$.

Let $\left\{Y_{n}, n \geq 1\right\}$ be a sequence of random variables. Let $S$ be a subset of natural number set $N$ and $F_{S}=\sigma\left(Y_{k}, k \in S\right)$. Define $\rho_{n}^{*}=\sup \{\operatorname{corr}(f, g):$ For all $S \times T \subset N \times N$, $\left.\operatorname{dist}(S, T) \geq n, \forall f \in L^{2}\left(F_{S}\right), g \in L^{2}\left(F_{T}\right)\right\}$, where

$$
\operatorname{corr}(f, g)=\frac{\operatorname{Cov}\left\{f\left(Y_{i}, i \in S\right), g\left(Y_{j}, j \in T\right)\right\}}{\left[\operatorname{Var}\left\{f\left(X_{i}, i \in S\right)\right\} \operatorname{Var}\left\{g\left(X_{j}, j \in T\right)\right\}\right]^{\frac{1}{2}}} .
$$

We call $\left\{Y_{n}, n \geq 1\right\}$ is a $\rho^{*}$-mixing sequence if

$$
\lim _{n \rightarrow \infty} \rho_{n}^{*}<1 \text {. }
$$

Let us note that, since $0 \leq \cdots \leq \rho_{n}^{*} \leq \rho_{n-1}^{*} \leq \cdots \leq \rho_{1}^{*} \leq 1,(1.2)$ is equivalent to

$$
\rho_{N}^{*}<1 \text { for some } N>1 \text {. }
$$

Bryc and Smolenski [3] and Peligrad and Gut [11] pointed out the importance of condition (1.2) in estimating the moments of partial sums or of maximum 
of partial sums. Various limit properties under the condition $\lim \rho_{n}^{*}<1$ were studied by Bradley [2] and Miller [10].

In this paper we shall extend Theorem $\mathrm{C}$ to the $\rho^{*}$-mixing case.

\section{Results}

The following lemma comes from Burton and Dehling [4].

Lemma 2.1. Let $\sum_{-\infty}^{\infty} a_{i}$ be an absolutely convergent series of real numbers with $a=\sum_{-\infty}^{\infty} a_{i}$ and $k \geq 1$. Then

$$
\lim _{n \rightarrow \infty} \frac{1}{n} \sum_{i=-\infty}^{\infty}\left|\sum_{j=i+1}^{i+n} a_{j}\right|^{k}=|a|^{k} .
$$

The following lemma will be useful. A proof appears in Peligrad and Gut [11].

Lemma 2.2. Let $\left\{Y_{n} ; n \geq 1\right\}$ be a sequence of $\rho^{*}$-mixing random variables with $E Y_{i}=0$ and $E\left|Y_{i}\right|^{q}<\infty$ for some $q \geq 2$. Assume that $\lim _{n \rightarrow \infty} \rho_{n}^{*}<1$. Then there exists a constant $C\left(q, N, \rho_{N}^{*}\right)$, depending on $q, N$, and $\rho_{N}^{*}$, with $N$ and $\rho_{N}^{*}$ defined via (1.3), such that

$$
E\left|S_{n}\right|^{q} \leq C\left(q, N, \rho_{N}^{*}\right)\left(\sum_{i=1}^{n} E\left|Y_{i}\right|^{q}+\left(\sum_{i=1}^{n} E Y_{i}^{2}\right)^{\frac{q}{2}}\right) \forall q \geq 2 .
$$

Our main result is as follows:

Theorem 2.3. Set $S_{n}=\sum_{k=1}^{n} X_{k}, n \geq 1$, where $\left\{X_{k} ; k \geq 1\right\}$ is defined as (1.1). Suppose that $\left\{Y_{i} ;-\infty<i<\infty\right\}$ is a sequence of identically distributed and $\rho_{n}^{*}$-mixing random variables with $E Y_{1}=0, E\left|Y_{1}\right|^{q}<\infty$ for some $q \geq 2$ and $\lim _{n \rightarrow \infty} \rho_{n}^{*}<1$ and that $\left\{a_{i} ;-\infty<i<\infty\right\}$ is a sequence of real numbers with $\sum_{i=-\infty}^{\infty}\left|a_{i}\right|<\infty$. Let $h(x)>0(x>0)$ be a slowly varying function and $1 \leq p<2, r>p$. Then $E\left|Y_{1}\right|^{r} h\left(\left|Y_{1}\right|^{p}\right)<\infty$ implies

$$
\sum_{n=1}^{\infty} n^{r / p-2-1 / p} h(n) E\left\{\left|S_{n}\right|-\epsilon n^{1 / p}\right\}^{+}<\infty \text { for all } \epsilon>0 .
$$

Remark. Let $a_{i+k}=1, i=k ; a_{i+k}=0, i \neq k, 1 \leq k \leq n$. Then $X_{k}=$ $Y_{k}, S_{n}=\sum_{k=1}^{n} X_{k}=\sum_{k=1}^{n} Y_{k}$. Hence Theorem 2.3 holds when $\left\{X_{k} ; k \geq 1\right\}$ is a sequence of identically distributed and $\rho^{*}$-mixing random variables.

Corollary 2.4. Under the conditions of Theorem 2.3 $E\left|Y_{1}\right|^{r} h\left(\left|Y_{1}\right|^{p}\right)<\infty$ implies

$$
\sum_{n=1}^{\infty} n^{r / p-2} h(n) P\left\{\left|S_{n}\right|>\epsilon n^{1 / p}\right\}<\infty \text { for all } \epsilon>0
$$


Proof. By Theorem 2.3 we have

$$
\begin{aligned}
& \sum_{n=1}^{\infty} n^{r / p-2-1 / p} h(n) E\left\{\left|S_{n}\right|-\epsilon n^{1 / p}\right\}^{+} \\
= & \sum_{n=1}^{\infty} n^{r / p-2-1 / p} h(n) \int_{0}^{\infty} P\left\{\left|S_{n}\right|-\epsilon n^{\frac{1}{p}}>x\right\} d x \\
= & \int_{0}^{\infty} \sum_{n=1}^{\infty} n^{r / p-2-1 / p} h(n) P\left\{\left|S_{n}\right|>(\epsilon+y) n^{\frac{1}{p}}\right\} n^{\frac{1}{p}} d y \\
= & \int_{0}^{\infty} \sum_{n=1}^{\infty} n^{r / p-2} h(n) P\left\{\left|S_{n}\right|>(\epsilon+y) n^{\frac{1}{p}}\right\} d y<\infty .
\end{aligned}
$$

Hence from (2.5) the result (2.4) follows.

\section{Proof of Theorem 2.3}

Recall that

where $a_{n i}=\sum_{k=1}^{n} a_{i+k}$.

$$
\sum_{k=1}^{n} X_{k}=\sum_{i=-\infty}^{\infty} \sum_{k=1}^{n} a_{i+k} Y_{i}=\sum_{i=-\infty}^{\infty} a_{n i} Y_{i},
$$

From Lemma 2.1, we can assume, without loss of generality, that

$$
\sum_{i=-\infty}^{\infty}\left|a_{n i}\right| \leq n, n \geq 1 \text { and } \tilde{a}=\sum_{i=-\infty}^{\infty}\left|a_{i}\right| \leq 1
$$

Let $S_{n}=\sum_{-\infty}^{\infty} a_{n i} Y_{i} I\left\{\left|a_{n i} Y_{i}\right| \leq x\right\}$. First note that for $x>n^{\frac{1}{p}}$,

$$
\begin{aligned}
x^{-1}\left|E S_{n}\right| & =x^{-1}\left|\sum_{i=-\infty}^{\infty} a_{n i} E Y_{i} I\left\{\left|a_{n i} Y_{i}\right|>x\right\}\right| \\
& \leq x^{-1} \sum_{i=-\infty}^{\infty}\left|a_{n i}\right| E\left|Y_{1}\right| I\left\{\left|a_{n i} Y_{1}\right|>x\right\} \\
& \leq x^{-1} n E\left|Y_{1}\right| I\left\{\tilde{a}\left|Y_{1}\right|>x\right\} \\
& \leq x^{-1} n E\left|Y_{1}\right| I\left\{\left|Y_{1}\right|>x\right\} \\
& \leq x^{-1} x^{p} E\left|Y_{1}\right| I\left\{\tilde{a}\left|Y_{1}\right|>x\right\} \\
& \leq E\left|Y_{1}\right|^{p} I\left\{\left|Y_{1}\right|>x\right\} \rightarrow 0 \text { as } x \rightarrow \infty .
\end{aligned}
$$

So, for $x$ large enough we have $x^{-1} E\left|S_{n}\right|<\epsilon / 2$. Then

$$
\begin{aligned}
& \sum_{n=1}^{\infty} n^{\frac{r}{p}-2-\frac{1}{p}} h(n) E\left\{\left|\sum_{k=1}^{n} X_{k}\right|-\epsilon n^{\frac{1}{p}}\right\}^{+} \\
= & \left.\sum_{n=1}^{\infty} n^{\frac{r}{p}-2-\frac{1}{p}} h(n) \int_{\epsilon n^{\frac{1}{p}}}^{\infty} P\left\{\left|\sum_{k=1}^{n} X_{k}\right| \geq x\right\} d x \text { (letting } x=\epsilon x^{\prime}\right)
\end{aligned}
$$




$$
\begin{aligned}
& =\sum_{n=1}^{\infty} n^{\frac{r}{p}-2-\frac{1}{p}} h(n) \epsilon \int_{n^{\frac{1}{p}}}^{\infty} P\left\{\left|\sum_{k=1}^{n} X_{k}\right| \geq \epsilon x^{\prime}\right\} d x^{\prime} \quad\left(\text { letting } x^{\prime}=x\right) \\
& \leq C \sum_{n=1}^{\infty} n^{\frac{r}{p}-2-\frac{1}{p}} h(n) \epsilon \int_{n^{\frac{1}{p}}}^{\infty}\left(P\left\{\sup _{i}\left|a_{n i} Y_{i}\right| \geq x\right\}+P\left\{\left|S_{n}-E S_{n}\right| \geq x \frac{\epsilon}{2}\right\}\right) d x \\
& =C \sum_{n=1}^{\infty} n^{\frac{r}{p}-2-\frac{1}{p}} h(n) \epsilon \int_{n^{\frac{1}{p}}}^{\infty}\left(I_{1}+I_{2}\right) d x
\end{aligned}
$$

where $I_{1}=P\left\{\sup _{i}\left|a_{n i} Y_{i}\right|>x\right\}$ and $I_{2}=P\left\{\left|S_{n}-E S_{n}\right| \geq x \frac{\epsilon}{2}\right\}$.

Set $I_{n j}=\left\{i \in \mathcal{I} ;(j+1)^{-\frac{1}{p}}<\left|a_{n i}\right| \leq j^{-\frac{1}{p}}\right\}, j=1,2, \ldots$ Then $\cup_{j \geq 1} I_{n j}=\mathcal{I}$. Note that (cf. Li et al. [7])

$$
\sum_{j=1}^{k} \# I_{n j} \leq n(k+1)^{\frac{1}{p}} .
$$

For $I_{1}$ and $1 \leq p<2, r \geq p$ noting that $E\left|Y_{1}\right|^{r} h\left(\left|Y_{1}\right|^{p}\right)<\infty$, we get

$$
\begin{aligned}
& \sum_{n=1}^{\infty} n^{\frac{r}{p}-2-\frac{1}{p}} h(n) \int_{n^{\frac{1}{p}}}^{\infty} I_{1} d x \\
\leq & C \sum_{n=1}^{\infty} n^{\frac{r}{p}-2-\frac{1}{p}} h(n) \int_{n^{\frac{1}{p}}}^{\infty} \sum_{i=-\infty}^{\infty} P\left\{\left|a_{n i} Y_{i}\right|>x\right\} d x \\
= & C \sum_{n=1}^{\infty} n^{\frac{r}{p}-2-\frac{1}{p}} h(n) \int_{n^{\frac{1}{p}}}^{\infty} \sum_{i=-\infty}^{\infty} P\left\{\left|a_{n i} Y_{1}\right|>x\right\} d x \\
\leq & C \sum_{n=1}^{\infty} n^{\frac{r}{p}-2-\frac{1}{p}} h(n) \int_{n^{\frac{1}{p}}}^{\infty} \sum_{j=1}^{\infty} \sum_{i \in I_{n j}} P\left\{\left|Y_{1}\right|>j^{\frac{1}{p}} x\right\} d x \\
\leq & C \sum_{n=1}^{\infty} n^{\frac{r}{p}-2-\frac{1}{p}} h(n) \int_{n^{\frac{1}{p}}}^{\infty} \sum_{j=1}^{\infty}\left(\# I_{n j}\right) \sum_{k \geq j x^{p}} P\left\{k \leq\left|Y_{1}\right|^{p}<k+1\right\} d x \\
\leq & C \sum_{n=1}^{\infty} n^{\frac{r}{p}-2-\frac{1}{p}} h(n) \int_{n^{\frac{1}{p}}}^{\infty} \sum_{k=\left[x^{p}\right]}^{\infty} \sum_{j=1}^{\left[k / x^{p}\right]}\left(\# I_{n j}\right) P\left\{k \leq\left|Y_{1}\right|^{p}<k+1\right\} d x \\
\leq & C \sum_{n=1}^{\infty} n^{\frac{r}{p}-2-\frac{1}{p}} h(n) \int_{n^{\frac{1}{p}}}^{\infty} \sum_{k=\left[x^{p}\right]}^{\infty} n\left(\frac{k}{x^{p}}+1\right)^{\frac{1}{p}} P\left\{k \leq\left|Y_{1}\right|^{p}<k+1\right\} d x \\
\leq & C \sum_{n=1}^{\infty} n^{\frac{r}{p}-1-\frac{1}{p}} h(n) \int_{n^{\frac{1}{p}}}^{\infty} \sum_{k=\left[x^{p}\right]}^{\infty} k^{\frac{1}{p}} x^{-1} P\left\{k \leq\left|Y_{1}\right|^{p}<k+1\right\} d x \\
\leq & C \int_{1}^{\infty} t^{\frac{r}{p}-1-\frac{1}{p}} h(t) \int_{t^{\frac{1}{p}}}^{\infty} \sum_{k=\left[x^{p}\right]}^{\infty} k^{\frac{1}{p}} x^{-1} P\left\{k \leq\left|Y_{1}\right|^{p}<k+1\right\} d x d t \\
& \left(\operatorname{letting} y=t^{\frac{1}{p}}\right)
\end{aligned}
$$




$$
\begin{aligned}
& \leq C \int_{1}^{\infty} y^{r-2} h\left(y^{p}\right) \int_{y}^{\infty} x^{-1} \sum_{k=\left[x^{p}\right]}^{\infty} k^{\frac{1}{p}} P\left\{k \leq\left|Y_{1}\right|^{p}<k+1\right\} d x d y \\
& \leq C \int_{1}^{\infty}\left(\int_{1}^{x} y^{r-2} h\left(y^{p}\right) d y\right) x^{-1} \sum_{k=\left[x^{p}\right]}^{\infty} k^{\frac{1}{p}} P\left\{k \leq\left|Y_{1}\right|^{p}<k+1\right\} d x \\
& \leq C \int_{1}^{\infty} x^{r-2} h\left(x^{p}\right) \sum_{k=\left[x^{p}\right]}^{\infty} k^{\frac{1}{p}} P\left\{k \leq\left|Y_{1}\right|^{p}<k+1\right\} d x \\
& \leq C \sum_{k=1}^{\infty} k^{\frac{1}{p}} P\left\{k \leq\left|Y_{1}\right|^{p}<k+1\right\} \int_{1}^{(k+1)^{\frac{1}{p}}} x^{r-2} h\left(x^{p}\right) d x \\
& \leq C \sum_{k=1}^{\infty} k^{\frac{1}{p}} P\left\{k \leq\left|Y_{1}\right|^{p}<k+1\right\}(k+1)^{\frac{r-1}{p}} h(k+1) \\
& \leq C \sum_{k=0}^{\infty}(k+1)^{\frac{r}{p}} h(k+1) P\left\{k \leq\left|Y_{1}\right|^{p}<k+1\right\} \\
& \leq C E\left|Y_{1}\right|^{r} h\left(\left|Y_{1}\right|^{p}\right)+1<\infty .
\end{aligned}
$$

Now we estimate $I_{2}$, for $1 \leq p<2, r>1+\frac{p}{2}$. By Lemma 2.2 and Markov's inequality, we have for $q \geq 2$

$$
\begin{aligned}
& P\left\{\left|S_{n}-E S_{n}\right| \geq \frac{\epsilon}{2} x\right\} \leq C x^{-q} E\left|S_{n}-E S_{n}\right|^{q} \\
\leq & C x^{-q}\left(\left(\sum_{i=-\infty}^{\infty} a_{n i}^{2} E Y_{1}^{2} I\left\{\left|a_{n i} Y_{1}\right| \leq x\right\}\right)^{q / 2}+\sum_{i=-\infty}^{\infty} E\left|a_{n i} Y_{1}\right|^{q} I\left\{\left|a_{n i} Y_{1}\right| \leq x\right\}\right) .
\end{aligned}
$$

Then

$$
\begin{aligned}
& \sum_{n=1}^{\infty} n^{\frac{r}{p}-2-\frac{1}{p}} h(n) \int_{n^{\frac{1}{p}}}^{\infty} I_{2} d x \\
\leq & C \sum_{n=1}^{\infty} n^{\frac{r}{p}-2-\frac{1}{p}} h(n) \int_{n^{\frac{1}{p}}}^{\infty} x^{-q}\left(\sum_{i=-\infty}^{\infty} a_{n i}^{2} E Y_{1}^{2} I\left\{\left|a_{n i} Y_{1}\right| \leq x\right\}\right)^{\frac{q}{2}} d x \\
& +\sum_{n=1}^{\infty} n^{\frac{r}{p}-2-\frac{1}{p}} h(n) \int_{n^{\frac{1}{p}}}^{\infty} x^{-q} \sum_{i=-\infty}^{\infty} E\left|a_{n i} Y_{1}\right|^{q} I\left\{\left|a_{n i} Y_{1}\right| \leq x\right\} d x \\
= & C \sum_{n=1}^{\infty} n^{\frac{r}{p}-2-\frac{1}{p}} h(n) \int_{n^{\frac{1}{p}}}^{\infty}\left(I_{3}+I_{4}\right) d x .
\end{aligned}
$$

If $q \geq 2$ is large enough such that $q\left(\frac{1}{p}-\frac{1}{2}\right)>\frac{r}{p}-1$, then for $I_{3}$ we get

$$
\sum_{n=1}^{\infty} n^{\frac{r}{p}-2-\frac{1}{p}} h(n) \int_{n^{\frac{1}{p}}}^{\infty} I_{3} d x
$$




$$
\begin{aligned}
& =\sum_{n=1}^{\infty} n^{\frac{r}{p}-2-\frac{1}{p}} h(n) \int_{n^{\frac{1}{p}}}^{\infty} x^{-q}\left(\sum_{i=-\infty}^{\infty} a_{n i}^{2} E Y_{1}^{2} I\left\{\left|a_{n i} Y_{1}\right| \leq x\right\}\right)^{\frac{q}{2}} d x \\
& \leq C \sum_{n=1}^{\infty} n^{\frac{r}{p}-2-\frac{1}{p}+\frac{q}{2}} h(n) \int_{n^{\frac{1}{p}}}^{\infty} x^{-q} d x=C \sum_{n=1}^{\infty} n^{\frac{r}{p}-2-q\left(\frac{1}{p}-\frac{1}{2}\right)} h(n)<\infty .
\end{aligned}
$$

For $I_{4}$ and $r \geq 2$ we get

$$
\begin{aligned}
& \sum_{n=1}^{\infty} n^{\frac{r}{p}-2-\frac{1}{p}} h(n) \int_{n^{\frac{1}{p}}}^{\infty} I_{4} d x \\
= & \sum_{n=1}^{\infty} n^{\frac{r}{p}-2-\frac{1}{p}} h(n) \int_{n^{\frac{1}{p}}}^{\infty} x^{-q} \sum_{i=-\infty}^{\infty} E\left|a_{n i} Y_{1}\right|^{q} I\left\{\left|a_{n i} Y_{1}\right| \leq x\right\} d x \\
\leq & C \sum_{n=1}^{\infty} n^{\frac{r}{p}-2-\frac{1}{p}} h(n) \int_{n^{\frac{1}{p}}}^{\infty} x^{-q} \sum_{j=1}^{\infty} \sum_{i \in I_{n j}} E\left|a_{n i} Y_{1}\right|^{q} I\left\{\left|a_{n i} Y_{1}\right| \leq x\right\} d x \\
\leq & C \sum_{n=1}^{\infty} n^{\frac{r}{p}-2-\frac{1}{p}} h(n) \int_{n^{\frac{1}{p}}}^{\infty} x^{-q} \sum_{j=1}^{\infty}\left(\# I_{n j}\right) j^{-\frac{q}{p}} E\left|Y_{1}\right|^{q} I\left\{\left|Y_{1}\right|^{p} \leq x^{p}(j+1)\right\} d x \\
\leq & C \sum_{n=1}^{\infty} n^{\frac{r}{p}-2-\frac{1}{p}} h(n) \int_{n^{\frac{1}{p}}}^{\infty} x^{-q} \sum_{j=1}^{\infty}\left(\# I_{n j}\right) j^{-\frac{q}{p}} \\
& \times \sum_{0 \leq k \leq(j+1) x^{p}} E\left|Y_{1}\right|^{q} I\left\{k \leq\left|Y_{1}\right|^{p}<k+1\right\} d x . \\
\leq & C \sum_{n=1}^{\infty} n^{\frac{r}{p}-2-\frac{1}{p}} h(n)\left[\int_{n^{\frac{1}{p}}}^{\infty} x^{-q} \sum_{j=1}^{\infty}\left(\# I_{n j}\right) j^{-\frac{q}{p}} \sum_{k=0}^{\left[2 x^{p}\right]} E\left|Y_{1}\right|^{q} I\left\{k \leq\left|Y_{1}\right|^{p}<k+1\right\} d x\right. \\
& \left.+\int_{n^{\frac{1}{p}}}^{\infty} x^{-q} \sum_{j=1}^{\infty}\left(\# I_{n j}\right) j^{-\frac{q}{p}} \sum_{k=\left[2 x^{p}\right]+1}^{\left[(j+1) x^{p}\right]} E\left|Y_{1}\right|{ }^{q} I\left\{k \leq\left|Y_{1}\right|^{p}<k+1\right\} d x\right] \\
= & C \sum_{n=1}^{\infty} n^{\frac{r}{p}-2-\frac{1}{p}} h(n) \int_{n^{\frac{1}{p}}}^{\infty}\left(I_{5}+I_{6}\right) d x .
\end{aligned}
$$

Note that for $q \geq 1$ and $m \geq 1$, we have

$$
\begin{aligned}
n & \geq \sum_{i=-\infty}^{\infty}\left|a_{n i}\right|=\sum_{j=1}^{\infty} \sum_{i \in I_{n j}}\left|a_{n i}\right| \geq \sum_{j=1}^{\infty}\left(\# I_{n j}\right)(j+1)^{-\frac{1}{p}} \\
& \geq \sum_{j=m}^{\infty}\left(\# I_{n j}\right)(j+1)^{-\frac{1}{p}} \geq \sum_{j=m}^{\infty}\left(\# I_{n j}\right)(j+1)^{-\frac{q}{p}}(m+1)^{\frac{q}{p}-\frac{1}{p}} .
\end{aligned}
$$

So

$$
\sum_{j=m}^{\infty}\left(\# I_{n j}\right) j^{-q / p} \leq C n m^{-(q-1) / p}
$$


If $r \geq 2$ and $q>r$, for $I_{5}$ we get

$$
\begin{aligned}
& \sum_{n=1}^{\infty} n^{\frac{r}{p}-2-\frac{1}{p}} h(n) \int_{n}^{\infty} I_{5} d x \\
& =\sum_{n=1}^{\infty} n^{\frac{r}{p}-2-\frac{1}{p}} h(n) \int_{n^{\frac{1}{p}}}^{\infty} x^{-q} \sum_{j=1}^{\infty}\left(\# I_{n j}\right) j^{-\frac{q}{p}} \sum_{k=0}^{\left[2 x^{p}\right]} E\left|Y_{1}\right|^{q} I\left\{k \leq\left|Y_{1}\right|^{p}<k+1\right\} d x \\
& \leq C \sum_{n=1}^{\infty} n^{\frac{r}{p}-2-\frac{1}{p}} h(n) n \int_{n^{\frac{1}{p}}}^{\infty} x^{-q} \sum_{k=0}^{\left[2 x^{p}\right]} E\left|Y_{1}\right|^{q} I\left\{k \leq\left|Y_{1}\right|^{p}<k+1\right\} d x \\
& \leq C \int_{1}^{\infty} t^{\frac{r}{p}-1-\frac{1}{p}} h(t) \int_{t^{\frac{1}{p}}}^{\infty} x^{-q} \sum_{k=0}^{\left[2 x^{p}\right]} E\left|Y_{1}\right|^{q} I\left\{k \leq\left|Y_{1}\right|^{p}<k+1\right\} d x d t \\
& \text { letting } t=y^{p} \\
& \leq C \int_{1}^{\infty} y^{r-2} h\left(y^{p}\right) \int_{y}^{\infty} x^{-q} \sum_{k=0}^{\left[2 x^{p}\right]} E\left|Y_{1}\right|^{q} I\left\{k \leq\left|Y_{1}\right|^{p}<k+1\right\} d x d y \\
& \leq C \int_{1}^{\infty}\left(\int_{1}^{x} y^{r-2} h\left(y^{p}\right) d y\right) x^{-q} \sum_{k=0}^{\left[2 x^{p}\right]} E\left|Y_{1}\right|^{q} I\left\{k \leq\left|Y_{1}\right|^{p}<k+1\right\} d x \\
& \leq C \int_{1}^{\infty} x^{r-1-q} h\left(x^{p}\right) \sum_{k=0}^{\left[2 x^{p}\right]} E\left|Y_{1}\right|^{q} I\left\{k \leq\left|Y_{1}\right|^{p}<k+1\right\} d x \\
& \leq C \sum_{k=1}^{\infty} E\left|Y_{1}\right|^{q} I\left\{k \leq\left|Y_{1}\right|^{p}<k+1\right\} \int_{\left(\frac{k}{2}\right)^{\frac{1}{p}}}^{\infty} x^{r-1-q} h\left(x^{p}\right) d x \\
& \leq C \sum_{k=1}^{\infty} E\left|Y_{1}\right|^{q} I\left\{k \leq\left|Y_{1}\right|^{p}<k+1\right\} k^{\frac{r-q}{p}} h(k) \\
& \leq C \sum_{k=1}^{\infty}(k+1)^{\frac{r}{p}} h(k+1) P\left\{k \leq\left|Y_{1}\right|^{p}<k+1\right\} \\
& \leq C E\left|Y_{1}\right|^{r} h\left(\left|Y_{1}\right|^{p}\right)+1<\infty \text {. } \\
& \text { If } r \geq 2 \text {, then for } I_{6}, 1 \leq p<2 \text {, and } p>1+\frac{p}{2} \text {, we also get }
\end{aligned}
$$

$$
\begin{aligned}
& \sum_{n=1}^{\infty} n^{\frac{r}{p}-2-\frac{1}{p}} h(n) \int_{n^{\frac{1}{p}}}^{\infty} I_{6} d x \\
= & \sum_{n=1}^{\infty} n^{\frac{r}{p}-2-\frac{1}{p}} h(n) \int_{n^{\frac{1}{p}}}^{\infty} x^{-q} \sum_{j=1}^{\infty}\left(\# I_{n j}\right) j^{-\frac{q}{p}} \sum_{k=\left[2 x^{p}\right]+1}^{\left[(j+1) x^{p}\right]} E\left|Y_{1}\right|^{q} I\left\{k \leq\left|Y_{1}\right|^{p}<k+1\right\} d x
\end{aligned}
$$




$$
\begin{aligned}
& \leq C \sum_{n=1}^{\infty} n^{\frac{r}{p}-2-\frac{1}{p}} h(n) \int_{n^{\frac{1}{p}}}^{\infty} x^{-q} \sum_{k=\left[2 x^{p}\right]+1}^{\infty} \sum_{j \geq\left[\frac{k}{x^{p}}\right]-1}\left(\sharp I_{n j}\right) j^{-\frac{q}{p}} E\left|Y_{1}\right|^{q} I\left\{k \leq\left|Y_{1}\right|^{q}<k+1\right\} d x \\
& \leq C \sum_{n=1}^{\infty} n^{\frac{r}{p}-2-\frac{1}{p}} h(n) \int_{n^{\frac{1}{p}}}^{\infty} x^{-q} \sum_{k=\left[2 x^{p}\right]+1}^{\infty} n\left(\frac{k}{x^{p}}\right)^{-\frac{q-1}{p}} E\left|Y_{1}\right|^{q} I\left\{k \leq\left|Y_{1}\right|^{p}<k+1\right\} d x \\
& \leq C \int_{1}^{\infty} t^{\frac{r}{p}-1-\frac{1}{p}} h(t) \int_{t^{\frac{1}{p}}}^{\infty} x^{-1} \sum_{k=\left[2 x^{p}\right]+1}^{\infty} k^{-\frac{q-1}{p}} E\left|Y_{1}\right|^{q} I\left\{k \leq\left|Y_{1}\right|^{p}<k+1\right\} d x d t
\end{aligned}
$$

letting $t=y^{p}$

$$
\begin{aligned}
& \leq C \int_{1}^{\infty} y^{r-2} h\left(y^{p}\right) \int_{y}^{\infty} x^{-1} \sum_{k=\left[2 x^{p}\right]+1}^{\infty} k^{-\frac{q-1}{p}} E\left|Y_{1}\right|^{q} I\left\{k \leq\left|Y_{1}\right|^{p}<k+1\right\} d x d y \\
& \leq C \int_{1}^{\infty}\left(\int_{1}^{x} y^{r-2} h\left(y^{p}\right) d y\right) x^{-1} \sum_{k=\left[2 x^{p}\right]+1}^{\infty} k^{-\frac{q-1}{p}} E\left|Y_{1}\right|^{q} I\left\{k \leq\left|Y_{1}\right|^{p}<k+1\right\} d x \\
& \leq C \int_{1}^{\infty} x^{r-2} h\left(x^{p}\right) \sum_{k=\left[2 x^{p}\right]+1}^{\infty} k^{-\frac{q-1}{p}} E\left|Y_{1}\right|^{q} I\left\{k \leq\left|Y_{1}\right|^{p}<k+1\right\} d x \\
& \leq C \sum_{k=1}^{\infty} k^{-\frac{q-1}{p}} E\left|Y_{1}\right|^{q} I\left\{k \leq\left|Y_{1}\right|^{p}<k+1\right\} \int_{0}^{\left(\frac{k}{2}\right)^{\frac{1}{p}}} x^{r-2} h\left(x^{p}\right) d x
\end{aligned}
$$$$
\leq C \sum_{k=1}^{\infty} k^{\frac{r-q}{p}} h(k) E\left|Y_{1}\right|^{q} I\left\{k \leq\left|Y_{1}\right|^{p}<k+1\right\}
$$$$
\leq C \sum_{k=1}^{\infty}(k+1)^{\frac{r}{p}} h(k+1) P\left\{k \leq\left|Y_{1}\right|^{p}<k+1\right\}
$$

$\leq C E\left|Y_{1}\right|^{r} h\left(\left|Y_{1}\right|^{p}\right)+1<\infty$.

So by (3.3) and (3.4) we get

$$
\sum_{n=1}^{\infty} n^{\frac{r}{p}-2-\frac{1}{p}} h(n) \int_{n^{\frac{1}{p}}}^{\infty} I_{4} d x<\infty
$$

for $r \geq 2$ and $q>r$.

Note that $r \geq 2, q>2$ and $q\left(\frac{1}{p}-\frac{1}{2}\right)>\frac{r}{p}-1$ imply $q>r$. Hence, (3.2) and (3.5) yield

$$
\sum_{n=1}^{\infty} n^{\frac{r}{p}-2-\frac{1}{p}} h(n) \int_{n^{\frac{1}{p}}}^{\infty} I_{2} d x<\infty
$$

for $r \geq 2$ and $q>2$ such that $q\left(\frac{1}{p}-\frac{1}{2}\right)>\frac{r}{p}-1$.

If $1+\frac{p}{2}<r<2$ and $q=2$, then (3.6) follows from (3.1) and (3.2) since $I_{3}=I_{4}$. Thus we have $\sum_{n=1}^{\infty} n^{\frac{r}{p}-2-\frac{1}{p}} h(n) E\left\{\left|S_{n}\right|-\epsilon n^{\frac{1}{p}}\right\}^{+}<\infty$ for all $\epsilon>0$. 
Acknowledgments. The authors thank the referee for careful reading and comments on this manuscript. This paper was published in remembrance of Chung Woon University sabbatical year.

\section{References}

[1] J. I. Baek, T. S. Kim, and H. Y. Liang, On the convergence of moving average processes under dependent conditions, Aust. N. Z. J. Statist. 45 (2003), 331-342.

[2] R. C. Bradley, Equivalent mixing conditions for random fields, Ann. Probab. 21 (1993), 1921-1926.

[3] C. W. Bryc and W. Smolenski, Moment conditions for almost sure convergence of weakly correlated random variables, Proc. Amer. Math. Soc. 119 (1993), 629-635.

[4] R. M. Burton and H. Dehling, Large deviation for some weakly dependent random process, Statist. Probab. Lett. 9 (1990), 397-401.

[5] Y. S. Chow, On the rate of moment convergence of sample sums and extremes, Bull. Inst. Math. Acad. Sinica 16 (1988), 177-201.

[6] I. A. Ibragimov, Some limit theorems for stationary processes, Theory Probab. Appl. 7 (1962), 349-382.

[7] D. L. Li, M. B. Rao, and X. C. Wang, Complete convergence of moving average processes, Statist. Probab. Lett. 14 (1992), 111-114.

[8] Y. X. Li and L. X. Zhang, Complete moment convergence of moving average processes under dependence assumptions, Statist. Probab. Lett. 70 (2004), 191-197.

[9] H. Y. Liang, Complete convergence for weighted sums of negatively associated random variables, Statist. Probab. Lett. 48 (2000), 317-325.

[10] C. Miller, Three theorems on $\rho^{*}$-mixing random fields, J. Theor. Probab. 7 (1994), 867-882.

[11] M. Peligrad and A. Gut, Almost sure results for a class of dependent random variables, J. Theor. Prob. 12 (1999), 87-104.

[12] L. X. Zhang, Complete convergence of moving average processes under dependence assumptions, Statist. Probab. Lett. 30 (1996), 165-170.

Mi-Hwa Ko

Institute of Basic Natural Science

WONKWANG UNIVERSITY

JEONBuK 570-749, KoreA

E-mail address: songhack@wonkwang.ac.kr

TAe-Sung KIM

Institute of Basic Natural Science

WONKWANG UNIVERSITY

JeONBUK 570-749, KoreA

E-mail address: starkim@wonkwang.ac.kr

Dae-Hee Ryu

Department of Computer Science

ChungWoon University

Chungnam 350-701, Korea

E-mail address: rdh@chungwoon.ac.kr 